\title{
Preparatory Activity in Occipital Cortex in Early Blind Humans Predicts Auditory Perceptual Performance
}

\author{
Alexander A. Stevens, Mathew Snodgrass, Daniel Schwartz, and Kurt Weaver \\ Department of Psychiatry, Oregon Health and Science University, Portland, Oregon 97239
}

\begin{abstract}
Early onset blindness leads to a dramatic alteration in the way the world is perceived, a change that is detectable in the organization of the brain. Several studies have confirmed that blindness leads to functional alterations in occipital cortices that normally serve visual functions. These reorganized brain regions respond to a variety of tasks and stimuli, but their specific functions are unclear. In sighted individuals, several studies have reported preparatory activity in retinotopic areas, which enhances perceptual sensitivity. "Baseline shifts," changes in activity associated with a cue predicting an upcoming event, provides a marker for attentional modulation. Here we demonstrate that, in early blind subjects, medial occipital areas produced significant blood oxygenation level-dependent (BOLD) responses to a cue signaling an auditory discrimination trial but not to a cue indicating a no-trial period. Furthermore, the amplitude of the BOLD response in the anterior calcarine sulcus of early blind subjects correlated with their discrimination performance on the auditory backward masking task. Preparatory BOLD responses also were present in auditory cortices, although they were more robust in blind than sighted control subjects. The pattern of response in visual areas is similar to preparatory effects observed during visual selective attention in sighted subjects and consistent with the hypothesis that the mechanisms implicated in visual attention continue to modulate occipital cortex in the early blind. A possible source of this top-down modulation may be the frontoparietal circuits that retain their connectivity with the reorganized occipital cortex and as a result influence processing of nonvisual stimuli in the blind.
\end{abstract}

Key words: blindness; attention; visual cortex; auditory cortex; auditory perception; fMRI

\section{Introduction}

The occipital cortex in early onset blind (EB) individuals responds to a broad class of stimuli from auditory and tactile domains and to a variety of cognitive tasks. For example, functional magnetic resonance imaging (fMRI) and positron emission tomography studies have established that medial occipital areas respond to a variety of stimuli and tasks (Sadato et al., 1998; De Volder et al., 1999; Weeks et al., 2000; Arno et al., 2001; Röder et al., 2002; Amedi et al., 2003; Burton, 2003; Burton et al., 2004; Gougoux et al., 2005; Poirier et al., 2005; Collignon et al., 2006). Indeed, the function of the "visual cortex" in early onset blindness remains speculative, precisely because it responds to so many different stimuli and tasks. What is clear is that EB subjects must attend to the stimuli to elicit responses in occipital cortex, particularly within the calcarine sulcus. Studies of early onset blindness have revealed alteration in the amplitudes and latencies of event-related potentials recorded over occipital electrodes under attended versus unattended conditions (Kujala et al., 1995, 2000; Liotti et al., 1998; Sadato et al., 1998), with similar effects localized to medial and lateral occipital areas using fMRI (Weaver

Received April 13, 2007; revised Aug. 20, 2007; accepted Aug. 23, 2007.

This work was supported by National Eye Institute Grant EY13584. We thank Xin Li and the other members of the Oregon Health and Science University Advanced Imaging Research Center for technical help. We also thank Arun Garg for insightful discussions on the study and manuscript.

Correspondence should be addressed to Dr. Alexander A. Stevens, Department of Psychiatry, Mail Code L470, Oregon Health and Science University, 3181 SW Sam Jackson Park Road, Portland, OR 97239-3098. E-mail: stevenal@ohsu.edu.

DOI:10.1523/JNEUROSCI.1669-07.2007

Copyright $\odot 2007$ Society for Neuroscience $\quad$ 0270-6474/07/2710734-08\$15.00/0 and Stevens, 2007). These results are consistent with selective attention modulation of the visual cortical areas.

Two mechanisms have been proposed to drive reorganization of visual cortex in early blindness (Burton et al., 2002; PascualLeone et al., 2005). First, crossmodal plasticity results from the persistence of auditory and somatosensory driven synapses that are usually pruned during development (Huttenlocher, 2002; Neville and Bavelier, 2002). Second, physiological mechanisms that are masked by the presence of visual processes may retain their functions in the reorganized cortex (Pascual-Leone and Hamilton, 2001; Burton et al., 2002). One possibility is that the mechanisms responsible for selective attention effects recorded within visual cortex in sighted control (SC) individuals persist in the blind and act on the crossmodally reorganized neural circuits. It may then be possible to detect attention-related functions in EB subjects that have been demonstrated in sighted individuals using cued visual attention paradigms (Kastner et al., 1999; Ress et al., 2000; Ruff and Driver, 2006; Taylor et al., 2007). Here we tested whether auditory cues that predicted auditory discrimination trials elicited a preparatory blood oxygenation level-dependent (BOLD) response activity in visual cortical fields in EB subjects during auditory discrimination tasks, similar to effects that have been reported in sighted individuals during visual tasks. A second issue we explored was whether preparatory effects were present in auditory cortices. EB individuals show a variety of enhanced auditory perceptual abilities compared with sighted control (Niemeyer and Starlinger, 1981; Muchnik et al., 1991), including on the auditory backward recognition masking (ABRM) task used in the 

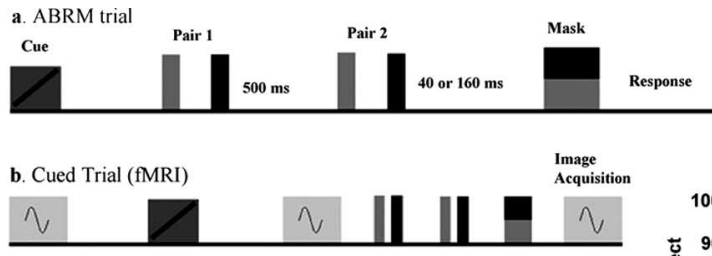

c. Trial-cue alone
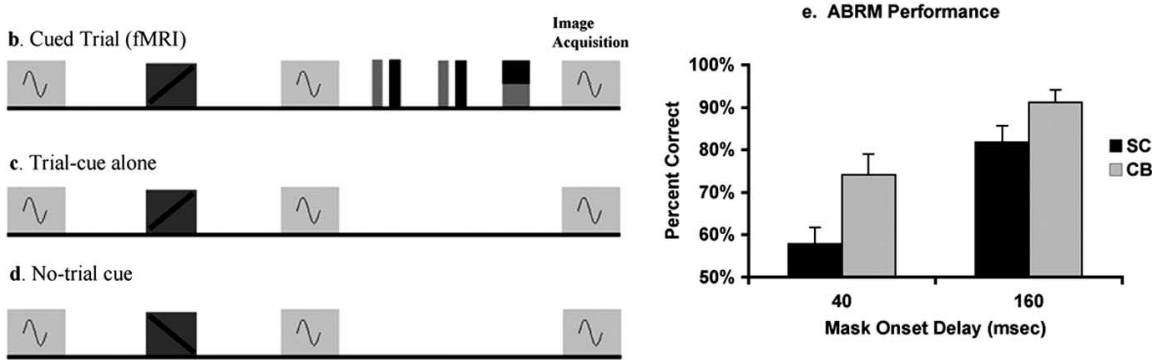

Figure 1. Schematic of the three trial conditions used in the fMRI study. $\boldsymbol{a}$, Auditory backward recognition masking trial structure. $\boldsymbol{b}$, During scanning, a trial cue (dark square with black line rising left to right) preceded the trial by one volume acquisition, and the trials were presented during the silent period between acquisitions. Trials were separated by three, four, five, or seven volume acquisitions. $\boldsymbol{c}, \boldsymbol{d}$, Trial-cue alone trials $(\boldsymbol{c})$ and no-trial cue $(\boldsymbol{d})$ (dark square with black line falling left to right) trials, were followed by a variable intertrial interval. $e$, EB and SC performance on the 40 and 160 ms mask delay conditions during scanning.

current study (Stevens and Weaver, 2005). Early blind individuals also show selective attention enhancements that may contribute to better auditory perceptual skills, yet few studies have reported alterations within auditory cortices despite these perceptual changes (cf. Elbert et al., 2002). Enhancements in auditory perception may be reflected in alteration in the BOLD signal in auditory cortices in the EB subjects compared with SC subjects.

\section{Materials and Methods}

Subjects. Twelve EB subjects (eight women; age, $47.3 \pm 10.5$ years; education, $17.5 \pm 3.4$ years) and 15 SC participants participated in the study. One SC subject's imaging data were discarded because of excessive signal spiking artifacts present in both functional scans. The resulting SC group consisted of 14 participants (six women; age, $51.2 \pm 7.1$ years; education, $17.8 \pm 2.9$ years). One functional scan of an EB participant was also discarded because of large signal spikes ( $>10 \%$ change) in the dataset. Eleven of the EB subjects reported having no light perception. One EB subject reported having minimal light perception but was unable to reliably distinguish when the overhead fluorescent lights were on or off in the testing room. Subjects' hand preferences were assessed by a handedness survey. Seven EB subjects were classified as right-handed, two lefthanded, and three mixed. All SC subjects were right-handed. Subjects were screened by self-report for drug or alcohol abuse in the last 5 years, major medical problems or neurological disorders that could affect cortical function, or the presence of medical devices or metal that could interfere with the scanner or pose a risk to the subject. This study was approved by the Oregon Health and Science University Institutional Review Board and conducted in accordance with National Institutes of Health human subject guidelines. All subjects provided informed consent; Braille forms were made available to the EB subjects.

Procedure. The procedure used for training subjects was analogous to that used in a previous behavioral study (Stevens and Weaver, 2005). Subjects were initially screened for hearing loss and then trained on temporal order judgment (TOJ) and ABRM tasks before scanning. The TOJ task is a measure of sensitivity to the order of two brief tones (Hirsch, 1959; Efron, 1963). The TOJ task was used to adjust the tone-pair stimuli used in the ABRM task compare temporal order discrimination thresholds for each subject. Behavioral training began with the TOJ threshold procedure to determine the smallest stimulus onset asynchrony (SOA) at which subjects could accurately indicate the order of presentation of a low $(2 \mathrm{kHz})$ and a high $(4 \mathrm{kHz})$ tone, each $10 \mathrm{~ms}$ in duration. The SOA was shortened by $10 \%$ after three correct responses and increased by $10 \%$ after one incorrect response. The procedure was repeated twice, and the threshold was calculated using the mean SOA value of the last four reversals of each run. Subjects whose TOJ threshold SOA was above $25 \mathrm{~ms}$ were excused from the study to maintain comparable performance between the EB and SC groups. Training continued with a two tonepair discrimination task (without a mask). The tone pairs were created by combining the low and high tones into a high-low pair and a lowhigh pair, with each subject's SOA threshold from the TOJ task plus $10 \mathrm{~ms}$ used as the within tone-pair SOA for the ABRM task (Stevens and Weaver, 2005). Subjects were required to perform at a minimum accuracy of $85 \%$ on the tone-pair discrimination. If they performed below that mark, an additional $5 \mathrm{~ms}$ was added to the SOA. All subjects met this criterion within three repetitions of 40 trials. The discrimination task was repeated a maximum of three times, and, if the subject failed to reach the accuracy cutoff, they were excused from the study. The adjusted SOA threshold for the tonepair stimuli was used in the ABRM task. Subjects were then trained on the ABRM task with mask onset delays of 40 and $160 \mathrm{~ms}$.

Auditory backward recognition mask task. The ABRM trials (Fig. 1) consisted of two tone pairs ( $75 \mathrm{~dB}$ sound pressure level) separated by a $300 \mathrm{~ms}$ interstimulus interval (offset to onset) and judged whether the order of the second tone pair was the same as the order of the first tone pair. Each tone pair consisted of the same low $(2.0 \mathrm{kHz})$ and high $(4.0 \mathrm{kHz})$ tones used in the TOJ task with the SOAs set to the individual's TOJ threshold plus $10 \mathrm{~ms}$. After the second tone pair, a $100 \mathrm{~ms}$ mask ( $80 \mathrm{~dB}$ sound pressure level) consisting of 2 and $4 \mathrm{kHz}$ ( $10 \mathrm{~ms}$ rise and fall time) was presented after a mask onset delay of 40 or $160 \mathrm{~ms}$ from the offset of the second tone pair. The mask onset delay was selected pseudorandomly such that either delay could occur on up to four consecutive trials.

Subjects underwent two functional scans while they performed the ABRM task (Fig. 1). The ABRM trials consisted of a trial cue (a $200 \mathrm{~ms}$ auditory "chirp") followed one repetition time (TR) later by an ABRM trial with a mask delay of either 40 or $160 \mathrm{~ms}$. Two additional trial conditions were also presented: (1) the trial-cue alone (TCA) condition in which a trial cue was presented with no subsequent ABRM trial, and (2) the no-trial cue (NTC) condition in which an auditory cue $(200 \mathrm{~ms}$ "chime") distinct from the trial cue was presented followed by a silent intertrial interval. Subjects were instructed that all ABRM trials would be preceded by the trial cue but, occasionally, the trial cue would be presented with no subsequent ABRM trial. They were also told that ABRM trials would never follow a no-trial cue. All stimuli were presented during the $1130 \mathrm{~ms}$ silent interval directly after the clustered acquisition of a given volume. Trials were separated with a jittered intertrial interval of three, four, five, or seven TRs (trial offset to cue onset) each occurring on $25 \%$ of the trials and balanced across conditions (cf. Garg et al., 2007). Within each scan, 80 ABRM trials and 40 trials each of the TCA and NTC conditions were presented.

All auditory stimuli were delivered via electrostatic headphones (Koss Corporation, Milwaukee, WI) mounted inside ear defenders (Howard Leight Industries, San Diego, CA) providing $26 \mathrm{~dB}$ (sound pressure level) of sound attenuation. Auditory stimuli were generated using a Sound Blaster Audigy 4 Pro soundcard (Creative Industries, Singapore) controlled by a personal computer running Presentation software (Neurobehavioral Systems, Albany, CA).

Functional MRI acquisition. All images were acquired with an echo planar imaging (EPI)-BOLD sequence on a whole-body 3T Siemens (Munich, Germany) Trio using a standard two-channel head coil. Functional data were acquired using a clustered volume acquisition to avoid interference from the scanner noise when presenting auditory stimuli. The functional images were acquired with the following parameters: TR, $2330 \mathrm{~ms}$; echo time (TE), $40 \mathrm{~ms}$; flip angle (FA), 90 ; field of view (FOV), $240 \mathrm{~mm}^{2}$; matrix, $64 \mathrm{~mm}^{2}$, resampled to $128 \mathrm{~mm}^{2}$. The volumes were acquired during the initial $1200 \mathrm{~ms}$ of each TR, leaving a $1130 \mathrm{~ms}$ silent interval during which trial stimuli were presented. The 17 slices with a 5 
$\mathrm{mm}$ slice thickness were oriented in an oblique-axial orientation for maximal coverage of cerebrum. Each functional scan consisted of 528 volume acquisitions and lasted $\sim 20 \mathrm{~min}$. Trials were not presented during the initial 10 acquisitions to allow subjects to acclimate to the scanner noise. A high-resolution, heavily T1-weighted magnetization-prepared rapid-acquisition gradient echo (MPRAGE) sequence was acquired for anatomic registration with the following functional volumes: $144 \mathrm{ob}-$ lique-axial slices; slice thickness, $1.0 \mathrm{~mm}$; TR, $2300 \mathrm{~ms}$; TE, $4 \mathrm{~ms}$; FA, 10; FOV, $240 \times 256 \mathrm{~mm}^{2}$; matrix, $256 \times 256 \mathrm{~mm}^{2}$. Subjects' heads were immobilized using padded clamps attached to the head coil.

Functional MRI analysis. All fMRI data analyses were processed using BrainVoyager QX (version 1.7; Brain Innovation, Maastricht, The Netherlands). Head motion in each EPI scan was corrected using a sixparameter rigid-body algorithm provided in the Siemens Syngo software. Temporal filtering was applied to the functional datasets including linear trend removal and a high-pass temporal filter (four cycles in time course). No spatial smoothing was applied. A slice scan-time correction was applied to temporally align all slices within each volume acquisition. Individual datasets were $z$ normalized to the mean signal intensity within each voxel across each scan, then aligned with the individual's highresolution (MPRAGE) anatomical brain scan, and resampled into $3.0 \times$ $3.0 \times 3.0 \mathrm{~mm}$ voxels. Each brain dataset was then convolved into a standard atlas coordinate system (Talairach and Tournoux, 1988) to allow the pooling of data across subjects.

Group parametric maps were calculated using a random effects model to allow a generalization of the results to the level of the population. Contrast maps were determined by comparing the $\beta$ weights calculated for each condition for thresholding each group using a $t$ statistic set to a value that yielded comparable probability thresholds for each group $(p<0.005$, uncorrected) adjusted for differences in the degrees of freedom in the denominator. These maps were then corrected for the probability of false-positive voxels using a spatial voxel-cluster threshold technique to produce a corrected $\alpha$ level with $p<0.05$ (Forman et al., 1995). This resulted in a cluster filter of $192 \mathrm{~mm}^{3}$ applied to the EB data, whereas the SC group required a cluster filter of $220 \mathrm{~mm}^{3}$ to achieve comparable false-positive probabilities.

Region of interest analysis. The functional MRI analyses were designed to identify those areas that responded during the ABRM trials and also produced a significantly greater response to the TCA condition than to the NTC condition. We identified these brain areas using the conjunction of the statistical maps: $[(160 \mathrm{~ms}+40 \mathrm{~ms}>$ baseline $) \cap($ TCA $>$ NTC)]. Group comparisons were performed on regions of interest (ROIs) identified by the conjunction analysis in and around the calcarine sulcus that were active in the EB group, as well as auditory cortical areas in the superior temporal plane in either EB or SC groups.

\section{Results}

\section{Behavioral performance}

Performance on the nonmasked version of the ABRM trials before scanning was also similar between the groups, although there was a trend toward better performance in the blind: (EB, $98.7 \pm$ $0.8 \%$; SC, $\left.95.4 \pm 1.4 \% ; t_{(24)}=1.81 ; p=0.08\right)$. The behavioral results of the ABRM trials during scanning revealed that $\mathrm{EB}$ subjects perform with greater accuracy than SC subjects on the ABRM task (40 ms trials: EB, $74 \pm 5 \%$; SC, $57 \pm 4 \%$; $160 \mathrm{~ms}$ trials: EB, $91 \pm 3 \%$; SC, $82 \pm 4 \%$ ) (Fig. 1). A repeated-measures ANOVA confirmed a significant effect of mask onset delay (40 and $160 \mathrm{~ms})$ on performance $\left(F_{(1,24)}=58.22 ; p<0.001\right)$, a result of the greater difficulty of the $40 \mathrm{~ms}$ condition, as well as a significant main effect of group, reflecting the greater discrimination performance of the $\mathrm{EB}\left(F_{(1,24)}=7.71 ; p=0.01\right)$. The group $\times$ condition interaction was not significant $\left(F_{(1,24)}=1.78 ; p=\right.$ $0.19)$.

\section{Functional MRI results}

The conjunction analysis $[(160 \mathrm{~ms}+40 \mathrm{~ms}>$ baseline $) \cap$ $($ TCA $>$ NTC)] used to isolate the specific preparatory response to the TCA condition revealed significant changes in the cortex in several brain regions of the EB subjects, including areas along the medial occipital cortex and auditory cortices (Table 1) (supplemental Fig. 1, available at www.jneurosci.org as supplemental material). In the SC group, the active voxels detected by the conjunction analysis were limited to right auditory cortical areas and the frontal cortex. To test for group differences and the specificity of the preparatory effects (i.e., differences between the TCA and NTC conditions), we analyzed the data from a subset of ROIs along the medial occipital wall in and around the calcarine sulcus. as well as in auditory cortices using a repeated-measures ANOVA, using within-subject factors of cue condition (TCA and NTC) and time (4.6 and $6.9 \mathrm{~s}$ ) and the between-subject factor of group (EB and SC). The analysis was restricted to the data acquired at 4.6 , and $6.9 \mathrm{~s}$ after the cue $(2.3$ and $4.6 \mathrm{~s}$ after the expected trial stimulus onset), in which the signals associated with the NTC and TCA conditions were most likely to diverge. The effect of interest reflecting a preparatory BOLD response that was specific to the EB group would appear as a three-way interaction of group, condition, and time.

\section{Occipital cortex}

Within the medial occipital lobe in the EB subjects, voxel clusters that had a greater response to the TCA than to NTC trials appeared in the anterior and posterior portion of the calcarine sulcus. Event-related responses for both $\mathrm{EB}$ and SC groups in these areas are shown in Figure 2. These medial occipital clusters were restricted almost exclusively to the right hemisphere in the $\mathrm{EB}$ group map. Additional areas that showed significant preparatory effects were present in the medial dorsal portion of the occipital cortex and extended up into the lateral surface of the posterior occipital lobe (Table 1). The BOLD response to the TCA condition produced a sustained positive event-related signal, whereas the NTC condition produced an initial small positive BOLD response followed by a sustained negative undershoot. In the anterior medial occipital ROI, there was a trend for the main effect of cue $\left(F_{(1,24)}=3.24 ; p=0.086\right)$, a main effect of time $\left(F_{(1,24)}=\right.$ $6.81 ; p=0.015)$, and no main effect of group $\left(F_{(1,24)}=1.39 ; p=\right.$ $0.252)$. There was a marginally reliable effect of condition $\times$ time $\left(F_{(1,24)}=4.17 ; p=0.052\right)$, no group $\times$ condition interaction $\left(F_{(1,24)}=1.78 ; p=0.204\right)$, and no group $\times$ time interaction $\left(F_{(1,24)}=0.78 ; p=0.387\right)$. However, the critical three-way interaction of condition $\times$ time $\times$ group was significant $\left(F_{(1,24)}=\right.$ $6.50 ; p=0.018$ ). This reflected the sustained response to the TCA condition compared with the deactivation in response to NTC present in the EB group (Fig. 3).

The same analysis was repeated in the posterior medial occipital ROI with similar results. The main effect of cue condition was reliable $\left(F_{(1,24)}=10.56 ; p=0.003\right)$, as was the main effect of time $\left(F_{(1,24)}=8.92 ; p=0.006\right)$ but not of group $\left(F_{(1,24)}=0.94 ; p=\right.$ $0.761)$. There was a reliable interaction of condition $X$ time $\left(F_{(1,24)}=7.71 ; p=0.010\right)$, a robust group $\times$ condition interaction $\left(F_{(1,24)}=17.65 ; p<0.000\right)$, but no group $\times$ time interaction $\left(F_{(1,24)}=0.72 ; p=0.406\right)$. However, the three-way interaction of condition $\times$ time $\times$ group was significant $\left(F_{(1,24)}=7.33 ; p=\right.$ 0.012 ).

\section{Correlations with behavioral performance}

Previous investigators have reported correlations between medial occipital activity and performance on verbal memory (Amedi et al., 2003; Raz et al., 2005) and auditory spatial discrimination (Gougoux et al., 2005). Therefore, we examined the two regions along the medial occipital cortex for correlation between each EB 
Table 1. Clusters of significantly greater activation $(p<0.05$, corrected) in the trial cue alone compared with the no-trial cue condition, Talairach coordinates of center of mass, and voxel cluster size

\begin{tabular}{|c|c|c|c|c|c|c|c|c|}
\hline \multirow[t]{2}{*}{ Brain region } & \multicolumn{4}{|c|}{ Early blind $(n=12)$} & \multicolumn{4}{|c|}{ Sighted control $(n=14)$} \\
\hline & $x$ & $y$ & $Z$ & Voxel \# & $x$ & $y$ & $Z$ & Voxel\# \\
\hline \multicolumn{9}{|l|}{ Occipital } \\
\hline$R$ cuneus & 9 & -80 & 21 & 1799 & & & & \\
\hline R cuneus & 17 & -87 & 5 & 1077 & & & & \\
\hline \multicolumn{9}{|l|}{ R temporal-occipital } \\
\hline junction & 45 & -66 & -3 & 447 & & & & \\
\hline R calcarine sulcus & 17 & -62 & 5 & 395 & & & & \\
\hline \multicolumn{9}{|l|}{ Temporal } \\
\hline L superior temporal sulcus & -50 & -50 & 7 & 790 & & & & \\
\hline L planum temporale & -51 & -30 & 8 & 300 & & & & \\
\hline R planum temoprale & 50 & -24 & 7 & 245 & 45 & -42 & 16 & 220 \\
\hline \multicolumn{9}{|l|}{ R superior temporal sul- } \\
\hline cus & 51 & -43 & 9 & 210 & & & & \\
\hline \multicolumn{9}{|l|}{ Parietal } \\
\hline R intraparietal sulcus & 27 & -59 & 38 & 8764 & & & & \\
\hline$R$ cingulate gyrus & 2 & -22 & 30 & 1654 & 2 & -28 & 25 & 225 \\
\hline L precuneus & -1 & -53 & 50 & 1078 & & & & \\
\hline L intraparietal sulcus & -30 & -53 & 41 & 1021 & & & & \\
\hline \multicolumn{9}{|l|}{ Frontal } \\
\hline R inferior frontal gyrus & 41 & 20 & 7 & 4191 & 29 & 22 & 9 & 569 \\
\hline L insula & -33 & 14 & 9 & 2991 & & & & \\
\hline$R$ cingulate gyrus & 6 & 13 & 39 & 2964 & & & & \\
\hline R middle frontal gyrus & 33 & 46 & 22 & 2475 & 38 & -2 & 37 & 979 \\
\hline R middle frontal gyrus & 39 & 30 & 33 & 462 & & & & \\
\hline R frontal eye field & 29 & -6 & 53 & 1284 & & & & \\
\hline L superior frontal gyrus & -18 & -6 & 57 & 474 & & & & \\
\hline L middle frontal gyrus & -29 & 38 & 30 & 290 & & & & \\
\hline R superior frontal gyrus & 0 & 18 & 49 & 257 & 5 & 29 & 45 & 222 \\
\hline L thalamus & -12 & -10 & 1 & 269 & & & & \\
\hline
\end{tabular}

R, Right; L, left.
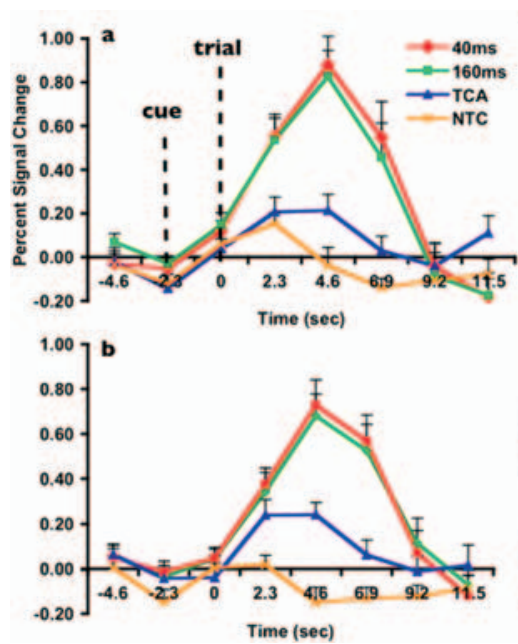

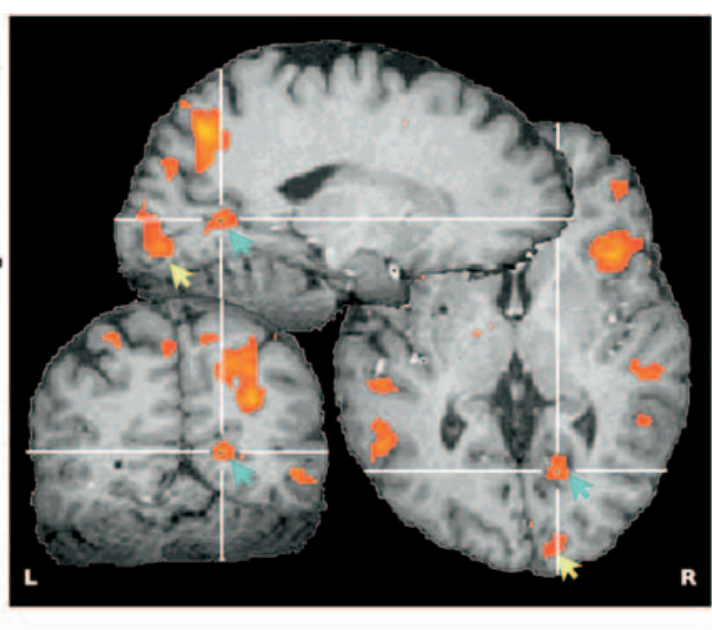

$\mathbf{R}$

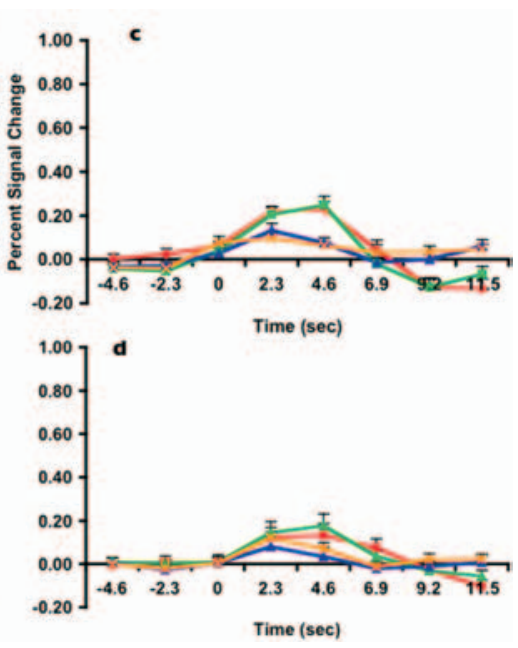

Figure 2. $\quad \boldsymbol{a}, \boldsymbol{b}$, Two regions of interest identified in the early onset blind subjects in the anterior ( $\boldsymbol{a}$ ) (blue arrows) and posterior ( $\boldsymbol{b}$ ) calcarine sulcus (yellow arrow) produced event-related BOLD response with a differential response to the TCA and NTC conditions. $\boldsymbol{c}, \boldsymbol{d}$, In contrast, the SC subjects had small event-related responses to both TCA and NTC conditions in the anterior (c) and posterior (d) calcarine sulcus. L, Left; R, right.

subjects' signal intensity at $4.6 \mathrm{~s}$ after onset for each trial condition (40 and $160 \mathrm{~ms}$, TCA and NTC) and performance on the ABRM trials. In the anterior calcarine sulcus, there was no significant correlation between the peak signal on the 40 or $160 \mathrm{~ms}$ conditions with performance accuracy on the corresponding trials (both Pearson's $r<0.21$, NS). However, the peak signal of the TCA trials was significantly positively correlated with average performance accuracy on the 40 and 160 ms trials (Pearson's $r_{(10)}$ $=0.57 ; p<0.05$ ) (Fig. 3). Conversely, the BOLD response from NTC trials were negatively correlated with average performance accuracy (Pearson's $r_{(10)}=-0.75 ; p<0.02$ ). No significant correlation between performance and any of the conditions was found in the posterior calcarine sulcus.

\section{Auditory cortices}

Active voxels showing baseline shifts in response to the TCA condition were identified bilaterally in auditory cortical areas in the EB group, including the lateral planum temporale (PT) and superior temporal sulcus (STS) (Fig. 4). In the SC group, significant voxel clusters were also present but limited to the right PT. 


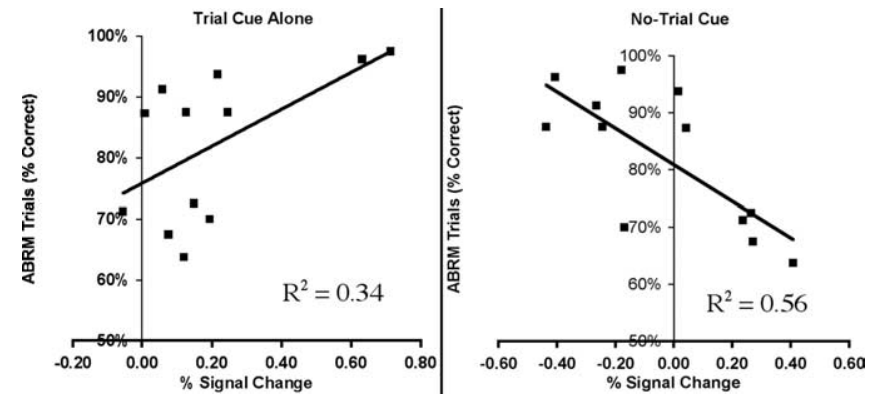

Figure 3. $\quad \boldsymbol{a}, \boldsymbol{b}$, Scatter plots and best-fit lines showing the correlation between early blind individuals' averaged performance on the 40 and $160 \mathrm{~ms}$ ABRM trials and percentage signal change $4.6 \mathrm{~s}$ after the expected trial onset for the trial-cue alone condition $(\boldsymbol{a})$ and the no-trial cue $(\boldsymbol{b})$.

The difference between the EB and SC subjects in auditory cortical areas (and most other brain areas outside the occipital lobe) was the lack of a differential response to the TCA and NTC conditions in the SC subjects (Fig. 3). This was reflected in the BOLD time course in the auditory cortical areas as well as in the repeated-measures ANOVAs. Analyses were performed on the ROIs constructed from active voxel clusters located in the superior temporal plane of the temporal lobes in the EB and SC groups. In the left PT, there was a main effect of cue $\left(F_{(1,24)}=\right.$ $5.95 ; p=0.022)$, a main effect of time $\left(F_{(1,24)}=15.41 ; p=0.001\right)$, but no main effect of group $\left(F_{(1,24)}=0.13 ; p=0.724\right)$. There was a reliable interaction of condition $\times$ time $\left(F_{(1,24)}=29.254 ; p<\right.$ $0.000)$, a reliable interaction of group $\times$ condition $\left(F_{(1,24)}=\right.$ $14.29 ; p=0.001)$, but no interaction of group $\times$ time $\left(F_{(1,24)}=\right.$ $0.04 ; p=0.854)$. However, there was a reliable three-way interaction of group $\times$ condition $\times$ time $\left(F_{(1,24)}=7.40 ; p=0.012\right)$, reflecting the differential response to the TCA and NTC in the EB group but a similar response to both cues in the SC (Fig. 4). In the right lateral PT, there was no significant main effect of cue $\left(F_{(1,24)}=1.04 ; p=0.318\right)$, but there was a reliable effect of time $\left(F_{(1,24)}=15.41 ; p=0.001\right)$ and of group $\left(F_{(1,24)}=6.40 ; p=\right.$ $0.018)$. The interaction of condition $\times$ time was significant $\left(F_{(1,24)}=15.49 ; p=0.001\right)$, but neither group $\times$ condition $\left(\mathrm{F}_{(1,24)}=2.34, p=0.139\right)$ nor group $\times$ time $\left(F_{(1,24)}=0.01 ; p=\right.$ $0.922)$ were significant. The three-way interaction of group $\times$ condition $\times$ time was marginally significant $\left(F_{(1,24)}=4.08 ; p=\right.$ 0.055), reflecting the differential response to the TCA and NTC in the EB group but a general response to both cues in the SC (Fig. 4 ). This reflected the sustained response to the TCA condition compared with the transient response to the NTC condition (Fig. 4).

In the right PT ROI, there was no main effect of cue condition $\left(F_{(1,24)}=2.37 ; p=0.137\right)$, time $\left(F_{(1,24)}=0.79 ; p=0.38\right)$, or group $\left(F_{(1,24)}=0.17 ; p=0.69\right)$. The interaction of condition $\times$ time was highly significant $\left(F_{(1,24)}=18.46 ; p<0.000\right)$, but no other two-way interaction approached significance. The threeway interaction of condition $\times$ time $\times$ group $(F<1, \mathrm{NS})$ also failed to reach significance, reflecting the presence of a stronger response to the TCA than NTC condition in both the EB and $\mathrm{SC}$ response within the right $\mathrm{PT}$ and superior temporal sulcus (Fig. 4).

There was no significant correlation (all Pearson's $r<0.35$ ) between performance on the ABRM and signal changes in any of the auditory cortical ROIs, in either EB or SC groups.

\section{Discussion}

Early blind individuals produced significant baseline shifts in the BOLD response in medial occipital areas to cues that indicated an upcoming discrimination trial. These responses were specific to cues signaling an ABRM trial as demonstrated by the absence of a preparatory response to a cue signaling that no trial would occur. This ruled out a general alerting effect. Although several researchers have noted attention modulation over occipital cortical areas in blind subjects measured under a variety of auditory and tactile stimulus conditions (Kujala et al., 1992; Röder et al., 1996; Liotti et al., 1998; Weaver and Stevens, 2007), to our knowledge, the present results provide the first evidence of a baseline shift in activity associated within the visual cortex in EB individuals.

The association between the magnitude of the BOLD response to the cues and individual subject performance further suggests that the observed hemodynamic activity was of functional significance to the discrimination. The EB individuals' peak BOLD responses in the anterior medial occipital area during the TCA condition were positively correlated with their performance on the ABRM task. Additionally, the BOLD response during the NTC condition correlated negatively with ABRM performance. Positive and negative BOLD responses in visual fields have been associated with modulation of local field potentials, and negative BOLD effects appear to contribute to contrast enhancement during visual tasks (Shmuel et al., 2002, 2006). The preparatory activity may reflect increased firing rates in neurons, which has been tied to increased sensitivity to subsequent targets (Reynolds et al., 1999; Ress et al., 2000). The negative correlation detected between the NTC response and ABRM performance suggests that, when relevant stimulus events are unlikely, hemodynamic activity decreases in the occipital areas in EB. This relationship between cue-mediated responses and performance suggests that selective attention mechanisms or conditional associations may contribute significantly to observed signals in the reorganized occipital cortex of EB individuals (Weaver and Stevens, 2007). Previous fMRI studies of EB subjects have reported correlations between the amplitude of signal changes in the medial occipital cortex during a semantic memory task (Amedi et al., 2003; Raz et al., 2005) and during a monaural sound localization task (Gougoux et al., 2005). The differences in the stimuli, task structure, and overall designs of those experiments suggest that some common factor may contribute to the association between the outcome measures and the BOLD signal observed in those experiments rather than the specific processes associated with the memory and discrimination tasks that were used. The observed effects within the medial occipital cortex of the EB suggest parallels to the anticipatory modulation of medial occipital cortex that has been observed in sighted subjects during cued visual discrimination tasks (Kastner et al., 1999; Ress et al., 2000). Recent neuronal recording studies of rat primary visual cortex have demonstrated that preparatory effects can be elicited by visual cues predicting reinforcement (Shuler and Bear, 2006). Also, fMRI studies have revealed that nonvisual cue stimuli evoke preparatory activity in human striate and extrastriate cortex even when the cues are indicative of nonvisual tasks (Jack et al., 2006). Both these findings indicate that conditional associations may be sufficient to elicit preparatory effects in early sensory cortices. The presence of conditional associations may be a critical precursor to selective attention effects.

Within auditory cortical areas, EB and SC groups produced significant preparatory effects in auditory cortices. These responses were generally more robust in the EB group, particularly 

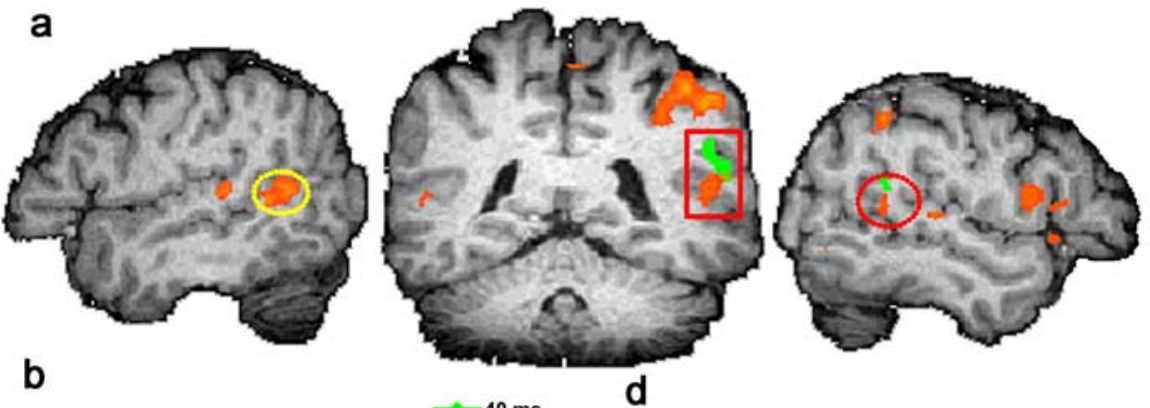

b

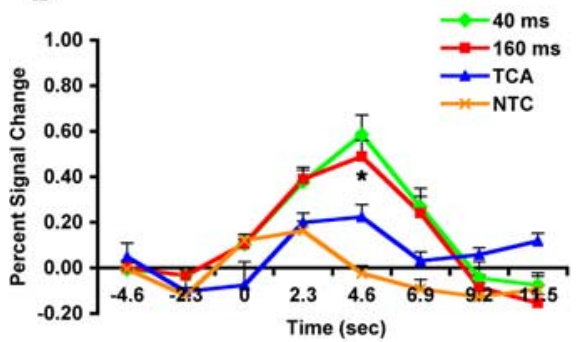

C

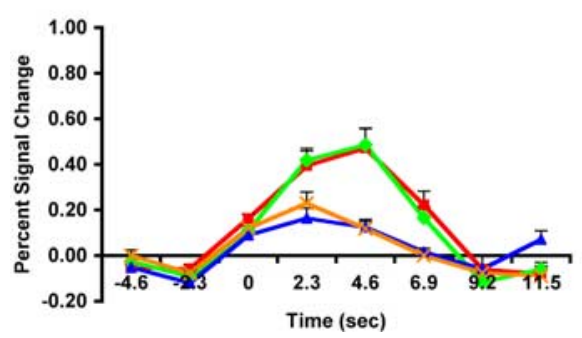

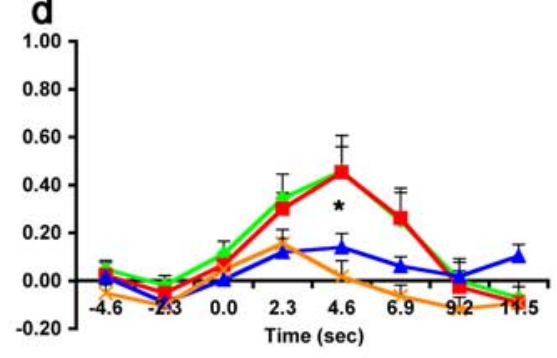

e

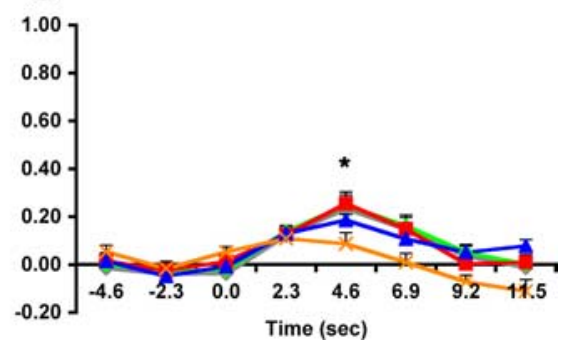

Figure 4. $\boldsymbol{a}$, Regions with significantly greater signal to the trial-cue alone than to the no-trial cue within the left and right temporal lobes in the early blind (orange) and sighted control (green) groups. $\boldsymbol{b}$ - $\boldsymbol{e}$, Event-related response plots for the left and right planum temporale and superior temporal sulcus in early blind $(\boldsymbol{b}, \boldsymbol{d})$ and sighted control $(\boldsymbol{c}, \boldsymbol{e})$ groups. ${ }^{*} p<0.05$.

shorter latencies may have reflected the process by which EB achieve more rapid stimulus processing (Röder et al., 1999a; Neville and Bavelier, 2002; Stevens and Weaver, 2005). Furthermore, the posterior shift in the distribution of the evoked potentials indicated that recruitment of posterior temporal, parietal, and occipital cortices may have contributed to the enhancement. Elbert et al. (2002) argued that expansion of the tonotopic maps in primary auditory cortex in blind individuals may account for some of the auditory perceptual advantages. Psychoacoustic studies have demonstrated that EB subjects outperform SC subjects on auditory spatial (Rice, 1969, Röder et al., 1999b), spectral (Niemeyer and Starlinger, 1981), and temporal (Muchnik et al., 1991; Stevens and Weaver, 2005) discrimination tasks, as well as on more complex attention and memory tasks (Röder and Rosler, 2003; Collignon et al., 2006). The widespread preparatory activity in the EB subjects within auditory cortical areas may reflect biasing effects that could contribute to their superiority in a range of auditory perceptual and cognitive abilities.

The reorganization of visual cortices in the blind may reflect some combination of de novo crossmodal plasticity interacting with normal neural circuitry of the occipital regions during development (PascualLeone and Hamilton, 2001; Burton, 2003;

in the left temporal lobe. Although both EB and SC subjects showed differential responding to the TCA and NTC cues in the right posterior PT (Fig. 4), differences between the EB and SC subjects were apparent in the left STS and lateral PT. This reflected a differential response to the TCA and NTC conditions in the EB that was absent in the SC. The differences between the groups were not attributable to an absence of BOLD response in the left auditory cortical areas in SC subjects but rather the lack of a differential response to the TCA and NTC, which was detected in the EB subjects. No significant correlation was detected between the hemodynamic responses in the auditory cortices and ABRM performance for either the EB or SC subjects, and therefore it is unclear whether the differences observed between $\mathrm{EB}$ and SC in the left STS is in some way responsible for the better performance of the EB subjects on the ABRM task. The current study design lacked an acoustically matched control condition to isolate the spectral and temporal components of the ABRM task. Although that was not the purpose of the current investigation, it nevertheless limits the interpretability of the responses to the ABRM trials within auditory cortices. Nonetheless, the detection of differential responding to the TCA and NTC conditions in the auditory cortices is important given the few studies that have reported differences in auditory cortices between blind and sighted individuals despite a broad range of enhanced auditory perceptual functions in EB individuals (Röder et al., 1999a; Elbert et al., 2002). Röder et al. (1999a) reported that an early negative evoked potential, the N1-evoked response, to standard stimuli in an auditory oddball design had shorter latencies and larger amplitudes in EB subjects than those of matched SC subjects. These
Pascual-Leone et al., 2005). The auditory cued preparatory effects in visual cortical fields in EB subjects are consistent with this position and resemble baseline shifts that have been observed in sighted individuals in response to cues signaling visual targets (Kastner et al., 1999; Ress et al., 2000). However, the source driving these preparatory effects in the occipital cortex in EB is unclear. Under normal visual development, nonhuman primate electrophysiological and anatomical studies (Barone et al., 2000; Buschman and Miller, 2007) and transcranial magnetic stimulation studies in humans suggest that the modulatory signals arise from frontoparietal systems and, in particular, the frontal eye fields (FEFs) (Connolly et al., 2002; Corbetta and Schulman, 2002; Ruff and Driver, 2006; Ruff et al., 2006; Taylor et al., 2007). As noted in Table 1, the TCA trial evoked a significant response in the EB subjects in several frontal and parietal areas, including the FEFs. Also, EB and SC individuals produced comparable responses in FEFs to endogenous cues during an auditory attention spatial task, suggesting that the network of brain regions that are associated with visual attention tasks may persist in EB subjects (Garg et al., 2007). However, accumulating evidence has suggested that FEF may have broader amodal functions. For instance, FEF produces BOLD responses in nonspatial crossmodal attention switching tasks (Shomstein and Yantis, 2004) and auditory spatial and nonspatial attention tasks (Opitz et al., 2002; Shomstein and Yantis, 2006), suggesting a role in allocating attention to stimuli of interest, regardless of spatial or modality characteristics.

Another source of occipital cortical modulation may arise from inferior frontal and temporal lobe networks associated with 
auditory selective attention. These networks, which responded to the TCA condition in both EB and SC subjects (Table 1), have been consistently detected in auditory attention tasks (Lipschutz et al., 2002; Opitz et al., 2002; Voisin et al., 2006). Additionally, Coull and coworkers have reported a network of frontal and parietal areas involved in orienting attention in time that overlap with both the inferior and dorsal frontal and parietal areas associated with auditory and visual attention (Coull and Nobre, 1998; Coull et al., 2003, 2004). These results demonstrate that multiple pathways influence baseline shifts in sensory cortical activity and may shape the reorganization of the occipital cortex in EB. Establishing the functional relationship between the reorganized occipital cortical areas and the neural sources of the preparatory signals in these areas will further understanding of mechanisms driving cortical reorganization and plasticity in response to sensory deprivation.

\section{References}

Amedi A, Raz N, Pianka P, Malach R, Zohary E (2003) Early "visual" cortex activation correlates with superior verbal memory performance in the blind. Nat Neurosci 6:758-766.

Amedi A, Floel A, Knecht S, Zohary E, Cohen LG (2004) Transcranial magnetic stimulation of the occipital pole interferes with verbal processing in blind subjects. Nat Neurosci 7:1266-1270.

Arno P, De Volder AG, Vanlierde A, Wanet-Defalque MC, Streel E, Robert A, Sanabria-Bohórquez S, Veraart C (2001) Occipital activation by pattern recognition in the early blind using auditory substitution for vision. NeuroImage 13:632-645.

Barone P, Batardiere A, Knoblauch K, Kennedy H (2000) Laminar distribution of neurons in extrastriate areas projecting to visual areas V1 and V4 correlates with the hierarchical rank and indicates the operation of a distance rule. J Neurosci 20:3263-3281.

Burton H (2003) Visual cortex activity in early and late blind people. J Neurosci 23:4005-4011.

Burton H, Snyder AZ, Diamond J, Raichle ME (2002) Adaptive changes in early and late blind: a fMRI study of verb generation to heard nouns. J Neurophysiol 88:3359-3371.

Burton H, Sinclair RJ, McLaren DG (2004) Cortical activity to vibrotactile stimulation: an fMRI study in blind and sighted individuals. Hum Brain Mapp 23:210-228.

Buschman TJ, Miller EK (2007) Top-down versus bottom-up control of attention in the prefrontal and posterior parietal cortices. Science 315:1860-1862.

Collignon O, Renier L, Bruyer R, Tranduy D, Veraart C (2006) Improved selective and divided spatial attention in early blind subjects. Brain Res 1075:175-182.

Connolly JD, Goodale MA, Menon RS, Munoz DP (2002) Human fMRI evidence for the neural correlates of preparatory set. Nat Neurosci 5:1345-1352.

Corbetta M, Shulman GL (2002) Control of goal-directed and stimulusdriven attention in the brain. Nat Rev Neurosci 3:201-215.

Coull JT, Nobre AC (1998) Where and when to pay attention: the neural systems for directing attention to spatial locations and to time intervals as revealed by both PET and fMRI. J Neurosci 18:7426-7435.

Coull JT, Walsh V, Frith CD, Nobre AC (2003) Distinct neural substrates for visual search amongst spatial versus temporal distractors. Brain Res Cogn Brain Res 17:368-379.

Coull JT, Vidal F, Nazarian B, Macar F (2004) Functional anatomy of the attentional modulation of time estimation. Science 303:1506-1508.

De Volder AG, Catalan-Ahumada M, Robert A, Bol A, Labar D, Coppens A, Michel C, Veraart C (1999) Changes in occipital cortex activity in early blind humans using a sensory substitution device. Brain Res 826:128-134.

Efron R (1963) Temporal perception, aphasia and deja vu. Brain Cogn 86:403-424.

Elbert T, Sterr A, Rockstroh B, Pantev C, Muller MM, Taub E (2002) Expansion of the tonotopic area in the auditory cortex of the blind. J Neurosci 22: 9941-9944.

Forman SD, Cohen JD, Fitzgerald M, Eddy WF, Mintun MA, Noll DC (1995) Improved assessment of significant activation in functional magnetic res- onance imaging (fMRI): use of a cluster-size threshold. Magn Reson Med 33:636-647.

Garg A, Schwartz D, Stevens AA (2007) Orienting auditory spatial attention engages frontal eye fields and medial occipital cortex in congenitally blind humans. Neuropsychologia 45:2307-2321.

Gougoux F, Zatorre RJ, Lassonde M, Voss P, Lepore F (2005) A functional neuroimaging study of sound localization: visual cortex activity predicts performance in early-blind individuals. PLoS Biol 3:e27.

Hirsch I (1959) Auditory perception of temporal order. J Acoust Soc Am 31:759-767.

Huttenlocher PR (2002) Neural plasticity. Cambridge, MA: Harvard UP.

Jack AI, Shulman GL, Snyder AZ, McAvoy M, Corbetta M (2006) Separate modulations of human V1 associated with spatial attention and task structure. Neuron 51:135-147.

Kastner S, Pinsk MA, De Weerd P, Desimone R, Ungerleider LG (1999) Increased activity in human visual cortex during directed attention in the absence of visual stimulation. Neuron 22:751-761.

Kujala T, Alho K, Paavilainen P, Summala H, Naatanen R (1992) Neural plasticity in processing of sound location by the early blind: an eventrelated potential study. Electroencephalogr Clin Neurophysiol 84:469-472.

Kujala T, Huotilainen M, Sinkkonen J, Ahonen AI, Alho K, Hämäläinen MS, Ilmoniemi RJ, Kajola M, Knuutila JE, Lavikainen J, Salonen O, Simola J, Standertskjöld-Nordenstam C, Tiitinen H, Tissari SO, Näätänen R (1995) Visual cortex activation in blind humans during sound discrimination. Neurosci Lett 183:143-146.

Kujala T, Alho K, Naatanen R (2000) Cross-modal reorganization of human cortical functions. Trends Neurosci 23:115-120.

Liotti M, Ryder K, Woldorff MG (1998) Auditory attention in the congenitally blind: where, when and what gets reorganized? NeuroReport 9:1007-1012.

Lipschutz B, Kolinsky R, Damhaut P, Wikler D, Goldman S (2002) Attention-dependent changes of activation and connectivity in dichotic listening. NeuroImage 17:643-656.

Muchnik C, Efrati M, Nemeth E, Malin M, Hildesheimer M (1991) Central auditory skills in blind and sighted subjects. Scand Audiol 20:19-23.

Neville H, Bavelier D (2002) Human brain plasticity: evidence from sensory deprivation and altered language experience. Prog Brain Res 138:177-188.

Niemeyer W, Starlinger I (1981) Do the blind hear better? Investigations on auditory processing in congenital or early acquired blindness. II. Central functions. Audiology 20:510-515.

Opitz B, Rinne T, Mecklinger A, von Cramon DY, Schroger E (2002) Differential contribution of frontal and temporal cortices to auditory change detection: fMRI and ERP results. NeuroImage 15:167-174.

Pascual-Leone A, Hamilton RH (2001) The metamodal organization of the brain. Prog Brain Res 134:427-445.

Pascual-Leone A, Amedi A, Fregni F, Merabet LB (2005) The plastic human brain cortex. Annu Rev Neurosci 28:377-401.

Poirier C, Collignon O, Devolder AG, Renier L, Vanlierde A, Tranduy D, Scheiber C (2005) Specific activation of the V5 brain area by auditory motion processing: an fMRI study. Brain Res Cogn Brain Res 25:650658.

Raz N, Amedi A, Zohary E (2005) V1 activation in congenitally blind humans is associated with episodic retrieval. Cereb Cortex 15:1459-1468.

Ress D, Backus BT, Heeger DJ (2000) Activity in primary visual cortex predicts performance in a visual detection task. Nat Neurosci 3:940-945.

Reynolds JH, Chelazzi L, Desimone R (1999) Competitive mechanisms subserve attention in macaque areas V2 and V4. J Neurosci 19:1736-1753.

Rice CE (1969) Perceptual enhancement in the early blind? Psychol Rec 19:1-14.

Röder B, Rosler F (2003) Memory for environmental sounds in sighted, congenitally blind and late blind adults: evidence for cross-modal compensation. Int J Psychophysiol 50:27-39.

Röder B, Rosler F, Hennighausen E, Nacker F (1996) Event-related potentials during auditory and somatosensory discrimination in sighted and blind human subjects. Brain Res Cogn Brain Res 4:77-93.

Röder B, Teder-Salejarvi W, Sterr A, Rosler F, Hillyard SA, Neville HJ (1999a) Improved auditory spatial tuning in blind humans. Nature 400:162-166.

Röder B, Rosler F, Neville HJ (1999b) Effects of interstimulus interval on 
auditory event-related potentials in congenitally blind and normally sighted humans. Neurosci Lett 264:53-56.

Röder B, Stock O, Bien S, Neville H, Rosler F (2002) Speech processing activates visual cortex in congenitally blind humans. Eur J Neurosci 16: 930-936.

Ruff CC, Driver J (2006) Attentional preparation for a lateralized visual distractor: behavioral and fMRI evidence. J Cogn Neurosci 18:522-538.

Ruff CC, Blankenburg F, Bjoertomt O, Bestmann S, Freeman E, Haynes JD, Rees G, Josephs O, Deichmann R, Driver J (2006) Concurrent TMSfMRI and psychophysics reveal frontal influences on human retinotopic visual cortex. Curr Biol 16:1479-1488.

Sadato N, Pascual-Leone A, Grafman J, Deiber MP, Ibanez V, Hallett M (1998) Neural networks for Braille reading by the blind. Brain 121:1213-1229.

Shmuel A, Yacoub E, Pfeuffer J, Van de Moortele PF, Adriany G, Hu X, Ugurbil K (2002) Sustained negative BOLD, blood flow and oxygen consumption response and its coupling to the positive response in the human brain. Neuron 36:1195-1210.

Shmuel A, Augath M, Oeltermann A, Logothetis NK (2006) Negative functional MRI response correlates with decreases in neuronal activity in monkey visual area V1. Nat Neurosci 9:569-577.
Shomstein S, Yantis S (2004) Control of attention shifts between vision and audition in human cortex. J Neurosci 24:10702-10706.

Shomstein S, Yantis S (2006) Parietal cortex mediates voluntary control of spatial and nonspatial auditory attention. J Neurosci 26:435-439.

Shuler MG, Bear MF (2006) Reward timing in the primary visual cortex. Science 311:1606-1609.

Stevens AA, Weaver K (2005) Auditory perceptual consolidation in earlyonset blindness. Neuropsychologia 43:1901-1910.

Talairach J, Tournoux P (1988) Co-planar stereotaxic atlas of the human brain. New York: Thieme.

Taylor PC, Nobre AC, Rushworth MF (2007) FEF TMS affects visual cortical activity. Cereb Cortex 17:391-399.

Voisin J, Bidet-Caulet A, Bertrand O, Fonlupt P (2006) Listening in silence activates auditory areas: a functional magnetic resonance imaging study. J Neurosci 26:273-278.

Weaver KE, Stevens AA (2007) Attention and sensory interactions within the occipital cortex in the early blind: an fMRI study. J Cogn Neurosci 19:1-16.

Weeks R, Horwitz B, Aziz-Sultan A, Tian B, Wessinger CM, Cohen LG, Hallett M, Rauschecker JP (2000) A positron emission tomographic study of auditory localization in the congenitally blind. J Neurosci 20:2664-2672. 